\title{
Staples Versus Sutures for Skin Closure in Standard Four Port Laparoscopic Cholecystectomy: A Prospective Cohort Study
}

Farhanul Huda ${ }^{1}$, Bhargav Gajula ${ }^{1}$, Sudhir Singh ${ }^{1}$, Shashank Kumar ${ }^{1}$, Manoj Joshua Lokavarapu ${ }^{1}$, Durga Sowmya ${ }^{1}$

1. General Surgery, All India Institute of Medical Sciences, Rishikesh, IND

Corresponding author: Bhargav Gajula, bhargavrayal@gmail.com

\section{Abstract \\ Introduction}

Many studies have been done comparing sutures versus skin staples in various wounds. To the author's knowledge, there is no study comparing these two in an laparoscopic cholecystectomy (LC) wound. Our study aims at comparing the clinical outcome of skin closure by monofilament nylon suture and stainlesssteel skin stapler in standard four-port LC. The results of this study can help in developing guidelines for skin closure in LC.

\section{Objective}

To compare the clinical outcome of skin closure by monofilament nylon suture and stainless-steel skin stapler in standard four-port LC.

\section{Methods}

The study was conducted as a time-bound prospective cohort study on diagnosed patients of cholelithiasis admitted in a single unit of the Department of Surgery at All India Institute of Medical Sciences, Rishikesh, India from February 2018 to February 2019. The standard four-port LC was done by the same surgeon. After the completion of the surgery, port closure was done using absorbable sutures, and skin was closed by either 2.0 monofilament nylon suture (Ethilon, Ethicon, Scotland) or stainless-steel staples (Proximate plus MD, 35W, Ethicon, Scotland). The time taken for skin closure in both the groups was noted using a stopwatch. Each wound was assessed on the post of day (POD) 1 during discharge, on POD 10 during suture/stapler removal, and POD 30 by the operating surgeon for pain, wound infection, scar status using validated scales.

\section{Statistical analysis used}

The outcome measures were calculated as mean and standard deviation. Continuous variables were analyzed using a two-tailed student t-test.

Review began 02/19/2021 Review ended 02/24/2021 Published 03/06/2021

(c) Copyright 2021 Huda et al. This is an open access article distributed under the terms of the Creative Commons Attribution License CC-BY 4.0., which permits unrestricted use, distribution, and reproduction in any medium, provided the original author and source are credited.

\section{Results}

Out of 48 suture vs 45 stapler cases the average time for closure is 277.14 seconds in suture vs 77.2 seconds $(p=0.0001)$ in the stapler group. All other parameters studied were not statistically significant among the two cohorts.

\section{Conclusion}

We conclude that stapler requires minimum time for closure with no statistically significant difference in wound infection, post-op pain, pain during removal, and scar results are the same in both the groups.

Categories: Pain Management, General Surgery, Quality Improvement

Keywords: laparoscopy, cholecystectomy, skin suture, staples, wound infection

\section{Introduction}

Laparoscopic cholecystectomy (LC) is the gold standard treatment for gallstone disease and is the most common laparoscopic surgery performed worldwide [1]. Surgical site wound closure plays a very important role in the postoperative success of any surgery. This importance is magnified with regards to LC in obtaining cosmesis. Wound complications are one of the most common causes of morbidity and mortality in laparoscopic procedures. Surgical wound closure aims to achieve rapid wound healing and a satisfactory cosmetic result, and also to reduce the risks of complications. Despite the large number of LC performed there is no agreed standard on the skin closure. Different methods and materials are used for wound closure. Skin closure of laparoscopic port entry wounds in LC is usually done with sutures. Staples are an alternative 
option to sutures. The potential advantage of staples is their low level of tissue reactivity thus reducing the local inflammatory response. It also reduces the width of the wound, time taken for wound closure, and the residual cross marks [2].

Many studies have been done comparing sutures versus skin staples in various wounds. To the author's knowledge, there is no study comparing these two in an LC wound. Our study aims at comparing the clinical outcome of skin closure by monofilament nylon suture and stainless steel skin stapler in standard four-port LC. The results of this study can help in developing guidelines for skin closure in LC.

\section{Materials And Methods}

The study was conducted as a time-bound prospective cohort study on diagnosed patients of cholelithiasis admitted in a single unit of the Department of Surgery at All India Institute of Medical Sciences, Rishikesh from February 2018 to February 2019. Informed consent was taken from each patient and the patients were randomized into the suture and the stapler group by computer randomization method. Each patient was screened as per the inclusion and exclusion criteria.

Patients with age $>18$ years and $<80$ years without comorbidities undergoing elective LC are included in the study. Patients with acute calculus cholecystitis, empyema gall bladder, immunocompromised patients, and LCs converted to open procedures were excluded from the study.

Standard preoperative preparation was followed in both the study groups. The LC was completed by the standard four-port technique by the same surgeon. After the completion of the surgery, the port closure was done in layers by absorbable sutures till the subcutaneous layer, and the skin was closed by either 2.0 monofilament nylon sutures (Ethilon, Ethicon, Scotland) by simple interrupted fashion, two stitches for 10 $\mathrm{mm}$ port and one stitch for $5 \mathrm{~mm}$ port or stainless-steel staples (Proximate plus MD, 35W, Ethicon, Scotland), three staples for $10 \mathrm{~mm}$ port and one staple for $5 \mathrm{~mm}$ port as per randomization. Each patient was given an I/V antibiotic according to the SAGES guidelines (single preoperative dose given within one hour of skin incision, and re-dosed if the procedure is more than 4 hours long). The time taken for skin closure in both groups was noted using a stopwatch. Each wound was assessed on the post of day (POD) 1 during discharge, on POD 10 during suture/stapler removal, and on POD 30 by the operating surgeon for pain, wound infection, and scar status using validated scales.

\section{Outcome measures}

Time taken for skin closure, rate of wound infection assessed by Southampton Grade [3], pain during suture \staple removal on POD1, 10, and 30 were evaluated by a numeric rating scale [4], the post-op scar was evaluated using the Stony Brook scar evaluation scale (SBSES) [5], rate of readmission, the requirement of secondary procedures like wound debridement, wound wash and resuturing were assessed for outcome measures.

\section{Statistical analysis}

Data was collected by a trained surgery resident and entered into an electronic database (MS Excel, Microsoft, Redmond, WA) and analyzed using statistical software SPSS (IBM, Armonk, NY, USA). The outcome measures were calculated as mean and standard deviation. Continuous variables were analyzed using a two-tailed student $t$-test. The results were reported as per the guidelines of the STROBE (Strengthening the Reporting of Observational Studies in Epidemiology) group [6].

\section{Results}

\section{Age}

The mean age of the suture group was 39.75 and that of the stapler was 38.4. The difference was not statistically significant with a p-value of 0.613 . Hence the study group was comparable in terms of age.

\section{Gender}

The majority of the study population were females comprising $79.57 \%$ and the rest $20.43 \%$ were males.

\section{Closure technique}

A total of 48 patients underwent skin suturing technique whereas 45 underwent stapler closure.

\section{The technique of wound closure and meantime of closure}

The mean time of closure in the suture group was 277.14 seconds and in the stapler group was 77.2 seconds. The difference was found to be statistically significant with $\mathrm{P}<0.001$; (95 CI - 166.5 to 233.3) (Table 1). 


\section{Cureus}

\begin{tabular}{|c|c|c|c|c|c|}
\hline Group & $\mathbf{N}$ & Mean Time (seconds) & Standard Deviation (SD) & T-value & P-value \\
\hline Suture & 48 & 277.1 & 96.9 & \multirow{2}{*}{1.618} & \multirow{2}{*}{0.0001} \\
\hline Stapler & 45 & 77.2 & 59.67 & & \\
\hline
\end{tabular}

TABLE 1: Results of mean time for wound closure among suture and stapler group.

The technique of wound closure and surgical site infection

Among the suture group, $83.3 \%$ and $95.8 \%$ had Grade 0 on POD1 and POD 30, respectively. Among the stapler group, 93.7\% and 97.7\% had Grade 0 on POD1 and POD30, respectively. None of the patients developed Grade 2 surgical site infection (SSI) in the stapler group and $8.3 \%$ of patients developed Grade 2 SSI in the suture group. However, these results were not statistically significant (Table 2).

\begin{tabular}{|c|c|c|c|c|c|}
\hline Group & $\begin{array}{l}\text { Southampton Grade on } \\
\text { Post Operative Day (POD) }\end{array}$ & $\begin{array}{l}\text { Normal Healing } \\
\text { (Grade 0), n (\%) }\end{array}$ & $\begin{array}{l}\text { Normal Healing with Mild } \\
\text { Erythema (Grade 1), } n(\%)\end{array}$ & $\begin{array}{l}\text { Erythema+ Other Signs of } \\
\text { Inflammation (Grade 2), } \mathrm{n} \\
(\%)\end{array}$ & $\begin{array}{l}\text { Serous Discharge or } \\
\text { Pus (Grade } 3 \text { and 4), n } \\
(\%)\end{array}$ \\
\hline \multirow{3}{*}{$\begin{array}{l}\text { Suture } \\
(\mathrm{n}= \\
48)\end{array}$} & POD1 & 40 (83.3) & 8 (16.6) & $0(0)$ & $0(0)$ \\
\hline & POD10* & 31 (64.5) & 9 (18.7) & $4(8.3)$ & $0(0)$ \\
\hline & POD30 & $46(95.8)$ & $2(4.1)$ & $0(0)$ & $0(0)$ \\
\hline \multirow{3}{*}{$\begin{array}{l}\text { Stapler } \\
(\mathrm{n}= \\
45)\end{array}$} & POD1 & 42 (93.7) & $3(6.6)$ & $0(0)$ & $0(0)$ \\
\hline & POD10 & 40 (88.8) & 5 (11.1) & $0(0)$ & $0(0)$ \\
\hline & POD30 & 44 (97.7) & 1 (2.2) & $0(0)$ & $0(0)$ \\
\hline
\end{tabular}

TABLE 2: Results of surgical site infection among suture and stapler group.

${ }^{*} n=44$; four patients were lost to follow-up on POD 10.

\section{Type of closure technique and numeric rating scale}

The mean numeric rating scale of the suture group was 1.64 and 1.10, for the stapler group it was 1.10 and 1.00 on POD 1 and POD 30 respectively without any statistical significance (Table 3).

\begin{tabular}{|c|c|c|c|c|}
\hline \multirow{4}{*}{ Numeric Rating Scale (NRS) } & Timing of Evaluation & Suture Mean Score (SD) & Stapler Mean Score (SD) & P-Value \\
\hline & POD1 & $1.64(1.76)$ & $1.46(1.35)$ & 0.596 \\
\hline & POD10 & $1.31(1.31)$ & $1.20(0.91)$ & 0.644 \\
\hline & POD30 & $1.10(0.87)$ & $1.00(0.70)$ & 0.532 \\
\hline
\end{tabular}

TABLE 3: Results of Numeric rating scale among the suture and stapler group.

Similarly, the mean Numeric Rating Scale (NRS) for pain during removal among sutures and staples was also not significant with a p-value of 0.501 .

\section{Type of closure technique and Stony Brook scar evaluation scale}

Mean SBSES for the suture group was 3.39 and 2.97, for the stapler group it was 3.06 and 3.02 on POD 1 and POD 30. The results were not comparable (Table 4). 


\section{Cureus}

\begin{tabular}{|l|l|l|l|l|}
\hline & Timing of Evaluation & Suture Mean Score (SD) & Stapler Mean Score (SD) & P-Value \\
\hline Stony Brook Scar Evaluation Scale (SBSES) & POD1 & $3.39(0.57)$ & $3.06(0.49)$ & 0.069 \\
& POD10 & $3.12(0.54)$ & $3.35(0.52)$ & 0.046 \\
& POD30 & $2.97(0.45)$ & $3.02(0.33)$ & 0.622 \\
\hline
\end{tabular}

TABLE 4: Results of the Stony Brook Scar Evaluation Scale among the suture and stapler group.

Type of closure technique and readmission, secondary procedures

None of the patients in both groups had required readmission or secondary procedures like wound debridement, re-suturing.

\section{Type of closure and cost}

The average cost of a single Ethilon suture (Code 664H) used in our study is INR 163 and of Proximate plus staple (PMW 35) for single-use is INR 1127.

\section{Discussion}

The present study shows a statistically significant difference in the time required to close the LC wound in the stapler group compared to the suture group consistent with other studies. But wound infection rate, pain during removal, and scar results were not statistically significant with the suture group.

\section{The technique of wound closure and meantime of closure}

Our study found that the staples have a shorter operative time, three times quicker compared to sutures. In addition to faster closure time, effective use of anesthetic gases by reducing anesthesia time and decreased likelihood of sustaining needlestick injury are the added advantages of using staples.

A study conducted on the pediatric scalp lacerations by Kanegaye et al. revealed that the staples were six times faster than the sutures without observed complications. The scar was cosmetically better and had less pain during removal in the stapler group. Hence, they concluded staple closure was safe, rapid, and costeffective [7]. Eldrup et al. evaluated 137 patients undergoing thoracic or abdominal surgery and concluded that the time for closure in the stapler group is less than conventional sutures. The closure with staples resulted in the additional expense, as the cost was 47 times higher than that of sutures with Dermalon [8].

The study done by Meiring et al. on 40 patients undergoing laparotomy revealed $80 \%$ time saving with staples but concluded that the final cost of the stapler has to be taken into account for its selection [9]. Ranaboldo and Rowe-jones compared skin stapler with subcuticular suture in 48 patients of laparotomy and found that the difference in time was significant, consistent with our study [10].

\section{The technique of wound closure and surgical site infection}

The author found no statistical difference in wound infection among the two groups. Tsujinaka et al., in a multicentric study, found no significant difference in wound infection between the subcuticular sutures and skin staples in 1080 patients undergoing skin closure after open gastrointestinal surgery [11]. However, some of the studies found an increased risk of wound infection in the staple groups in different types of operative procedures and varying incision lengths $[12,13]$. Tuuli et al. found a twofold risk of wound infection in the stapler group in comparison with subcuticular suture in a cesarean wound [12].

Chandrashekar et al. reported wound infection among the stapler group as $38.09 \%$ and the suture group was $16 \%$ in abdominal wound closure [13]. Basha et al. also found that stapler closure was attributed to an increased risk of wound infection. It led to a decrease in patient satisfaction, but it is not statistically significant to attribute stapler in decreased satisfaction [14].

\section{Type of closure technique and numeric rating scale}

Pain during stapler removal has varied opinions among available literature. Some studies reported that the staples have more pain during their removal $[15,16]$. One of the authors had suggested local application of eutectic mixture of lidocaine and prilocaine (EMLA) cream one hour prior to the removal of skin staples [17]. However, in the present study, there is no difference in pain perceived in both the groups after POD 1 and at the time of removal in accord with the findings of some studies [18-20]. 


\section{Type of closure technique and Stony Brook scar evaluation scale}

The poor and improper technique of suturing can lead to tension at the wound edges, whereas a stapler that can be applied equally on both the wound edges does not increase local tension and ischemia, thus can provide better cosmetic results [21]. Our study did not find any significant difference in post-operative scar status at a one-month follow-up. Cromi et al. found similar results in various skin closure methods in cesarean wounds and found equivalent cosmetic outcomes amongst closure methods [22].

\section{Type of closure and cost}

Usually, a single suture was sufficient to close all the laparoscopic port sites which is six times less costly than a skin staple. Our study is consistent with the results of Ranaboldo and Rowe-Jones where staple cost was five times greater than the sutures. Hence their study preferred the use of subcuticular suture [10].

The strengths of the study include the prospective nature of the study, the single surgeon operating and evaluating the cases which removes operator bias, use of validated methods for pain, wound, and scar assessment. Convenient sampling, time-bound cohort study, and small sample size are the limitations of our study. Hence more multicentric randomized studies are required to form a guideline for skin closure in LC wounds.

\section{Conclusions}

The surgical principles are evolving from open techniques to minimally invasive procedures and cosmesis is gaining the utmost importance expecting minimal scars in modern surgical practice. A good cosmetic scar not only gains satisfaction to the patient but also to the surgeon. The mandate to cosmesis is opening research options in search of ideal skin closure methods among conventional sutures, staples, steristrips, clips, and glue adhesives.

The results of our study found that the time taken for the closure of the LC port site wound with staples was three times faster than the suture group and saves operative time. However, there were no comparable results with other parameters studied. We recommend sutures for the LC port site skin closure without adding the additional cost of staples in resource-poor settings.

\section{Additional Information}

\section{Disclosures}

Human subjects: Consent was obtained or waived by all participants in this study. Institutional Ethics committee, All India Institute of Medical Sciences, Rishikesh issued approval 60/IEC/IM/2017. Institutional Ethics subcommittee reviewed and discussed your application dated (03/03/2017) to conduct the research study/protocol no "60/IEC/IM/2017" during the IEC meeting held on 04/10/2017 place AIIMS, Rishikesh. The project is ethically approved provisionally from subcommittee for further process. Animal subjects: All authors have confirmed that this study did not involve animal subjects or tissue. Conflicts of interest: In compliance with the ICMJE uniform disclosure form, all authors declare the following: Payment/services info: All authors have declared that no financial support was received from any organization for the submitted work. Financial relationships: All authors have declared that they have no financial relationships at present or within the previous three years with any organizations that might have an interest in the submitted work. Other relationships: All authors have declared that there are no other relationships or activities that could appear to have influenced the submitted work.

\section{References}

1. Acar T, Kamer E, Acar N, Atahan K, Bag H, Haciyanlı M, Akgül O: Laparoscopic cholecystectomy in the treatment of acute cholecystitis: comparison of results between early and late cholecystectomy. Pan Afr Med J. 2017, 26:49. 10.11604/pamj.2017.26.49.8359

2. Lal V, Shaikh TP, Narayan P, Ansari S, Deolekar S: Study of comparison between skin sutures and skin staplers: 400 case studies. Int J Res Med Sci. 2015, 3:277-281.

3. Tiwari S, Chauhan M, Shahapurkar VV, Akhtar MJ, Grover A, Prashad S, Nerkar E: Importance of Southampton wound grading system in surgical site infection. J Evol Med Dent Sci. 2014, 3:5491-5495. 10.14260/JEMDS/2014/2618

4. Downie WW, Leatham PA, Rhind VM, Wright V, Branco JA, Anderson JA: Studies with pain rating scales. Ann Rheum Dis. 1978, 37:378-381. 10.1136/ard.37.4.378

5. Fearmonti R, Bond J, Erdmann D, Levinson H: A review of scar scales and scar measuring devices . Eplasty. 2010, 10:e43.

6. Cuschieri S: The STROBE guidelines. Saudi J Anaesth. 2019, 13:31-34. 10.4103/sja.SJA_543_18

7. Kanegaye JT, Vance CW, Chan L, Schonfeld N: Comparison of skin stapling devices and standard sutures for pediatric scalp lacerations: a randomized study of cost and time benefits. J Pediatr. 1997, 130:808-813. 10.1016/s0022-3476(97)80025-x

8. Eldrup J, Wied U, Andersen B: Randomised trial comparing Proximate stapler with conventional skin closure. Acta Chir Scand. 1981, 147:501-502.

9. Meiring L, Cilliers K, Barry R, Nel CJ: A comparison of a disposable skin stapler and nylon sutures for wound 
closure. S Afr Med J. 1982, 62:371-372.

10. Ranaboldo CJ, Rowe-Jones DC: Closure of laparotomy wounds: skin staples versus sutures. Br J Surg. 1992, 79:1172-1173. 10.1002/bjs.1800791122

11. Tsujinaka T, Yamamoto K, Fujita J, et al.: Subcuticular sutures versus staples for skin closure after open gastrointestinal surgery: a phase 3, multicentre, open-label, randomised controlled trial. Lancet. 2013, 382:1105-1112. 10.1016/S0140-6736(13)61780-8

12. Tuuli MG, Rampersad RM, Carbone JF, Stamilio D, Macones GA, Odibo AO: Staples compared with subcuticular suture for skin closure after cesarean delivery: a systematic review and meta-analysis. Obstet Gynecol. 2011, 117:682-690. 10.1097/AOG.0b013e31820ad61e

13. Chandrashekar N, Prabhakar G, Shivakumarappa G, Tauheed F: A comparative study between skin sutures and skin staples in abdominal surgical wound closure. J Evol Med Dent Sci. 2013, 2:5180-5186.

14. Basha SL, Rochon ML, Quiñones JN, Coassolo KM, Rust OA, Smulian JC: Randomized controlled trial of wound complication rates of subcuticular suture vs staples for skin closure at cesarean delivery. Am J Obstet Gynecol. 2010, 203:1-8. 10.1016/j.ajog.2010.07.011

15. Stockley I, Elson RA: Skin closure using staples and nylon sutures: a comparison of results . Ann R Coll Surg Engl. 1987, 69:76-78.

16. George TK, Simpson DC: Skin wound closure with staples in the Accident and Emergency Department . J R Coll Surg Edinb. 1985, 30:54-56.

17. Banwell P, Deakin M, Holden J, Powell B: Removal of skin staples and the use of EMLA cream . Br J Plast Surg. 1997, 50:144-145. 10.1016/s0007-1226(97)91334-5

18. Batra J, Bekal RK, Byadgi S, Attresh G, Sambyal S, Vakade CD: Comparison of skin staples and standard sutures for closing incisions after head and neck cancer surgery: a double-blind, randomized and prospective study. J Maxillofac Oral Surg. 2016, 15:243-250. 10.1007/s12663-015-0809-y

19. Murphy M, Prendergast P, Rice J: Comparison of clips versus sutures in orthopaedic wound closure . Eur J Orthop Surg Traumatol. 2004, 14:16-18. 10.1007/s00590-003-0121-2

20. Oswal S, Borle R, Bhola N, Jadhav A, Surana S, Oswal R: Surgical staples: a superior alternative to sutures for skin closure after neck dissection-a single-blinded prospective randomized clinical study. J Oral Maxillofac Surg. 2017, 75:1-6. 10.1016/j.joms.2017.08.004

21. Eaton AC: A controlled trial to evaluate and compare a sutureless skin closure technique (Op-Site skin closure) with conventional skin suturing and clipping in abdominal surgery. Br J Surg. 1980, 67:857-860. 10.1002/bjs.1800671207

22. Cromi A, Ghezzi F, Gottardi A, Cherubino M, Uccella S, Valdatta L: Cosmetic outcomes of various skin closure methods following cesarean delivery: a randomized trial. Am J Obstet Gynecol. 2010, 203:1-8. 10.1016/j.ajog.2010.02.001 